\title{
Detection of Genital HPV Infection Using Urine Samples: a Population Based Study in India
}

\author{
Sasidharanpillai Sabeena ${ }^{1 *}$, Parvati Bhat ${ }^{2 *}$, Veena Kamath ${ }^{3}$, Mary Mathew $^{4}$, \\ Sushama Aswathyraj ${ }^{1}$, Santhosha Devadiga ${ }^{1}$, Suresha Prabhu ${ }^{1}$, Maity Hindol', \\ Akhil Chameetachal ${ }^{1}$, Anjana Krishnan ${ }^{1}$, Govindakarnavar Arunkumar ${ }^{1}$
}

\begin{abstract}
Background: Cervical cancer is the second commonest cancer among Indian women and its association with human papilloma virus (HPV) is well established. This preventable cancer accounts for the maximum number of cancer related deaths among rural Indian women. Unlike in developed countries there are no organized cervical cancer screening programmes in India due to lack of resources and manpower. Objective: To detect genital HPV infection using urine samples among asymptomatic rural women in the age group of 18-65 years. Materials and Methods: The study area chosen was Perdoor village in Udupi Taluk, Karnataka State and all the women in the age group of 18-65 years formed the study cohort. A cross sectional study was conducted by house visits and 1,305 women were enrolled in the study. After taking written informed consent a data sheet was filled and early stream random urine samples were collected, transported to a laboratory at $4^{\circ} \mathrm{C}$ and aliquoted. Samples were tested using nested HPV PCR with PGMY09/11 and GP5+/6+ primers. Positive cases were genotyped by sequence analysis. Results: Study participants included 1,134 sexually active and 171 unmarried women with a mean age at marriage of $22.1(\mathrm{SD}=3.9)$ years. Study area showed high female literacy rate of $86.6 \%$. Five urine samples tested positive for HPV DNA $(0.4 \%)$. Conclusions: We found very low genital HPV infection rate among women from monogamous community. This is the first major population based study carried out among asymptomatic rural women to detect genital HPV infectio from Karnataka using urine samples.
\end{abstract}

Keywords: Human papillomavirus - urine sample - asymptomatic women - Karnataka - nested PCR

Asian Pac J Cancer Prev, 17 (3), 1083-1088

\section{Introduction}

Eventhough cervical cancer is showing a declining trend in India it continues to be a major cause of cancer related mortality among Indian women. Worldwide cervical cancer is the fourth commonest cancer among women and the second commonest cancer occurring in Indian women. Association of Human Papillomavirus (HPV) with cervical cancer is well proven. Epidemiological studies on cervical cancer cases from India have found HPV positivity as high as 98\% (Das, 1992) (Bhatla et al., 2008). Persistent infection with high risk human papillomavirus is the most important risk factor for developing cervical cancer (Walboomers et al., 1999).

Cervical cancer is preventable by regular screening, vaccination, early detection and management of precancerous lesions. For decades Pap smear screening has played a vital role in cervical cancer prevention in the developed worlds. But this requires sophisticated health care infrastructure, trained cytopathologists, and multiple hospital visits which become an uphill task for women from developing nations. Unlike in developed countries there are no organized cervical cancer screening programmes in India due to lack of resources and manpower. Lack of awareness among healthy rural women regarding regular screening is another major hurdle in implementing such programmes. Last two decades witnessed the development of HPV molecular testing using cervical, vaginal and urine samples and US-FDA has approved HPV testing for cancer screening in 2014 which requires expertise and resources.

About $70 \%$ Indians live in villages and huge gap exists in the field of education, basic amenities and health care facilities between urban and rural areas. Even cancer incidence data published by major cancer registries is mainly collected from urban areas and medical colleges. Among healthy population HPV prevalence of 7-13\% reported according to Indian studies (Bruni et al., 2010). There is scarcity of data from rural India and Northeast

${ }^{I}$ Department of Virus Research, ${ }^{2}$ Department of Obstetrics and Gynecology, ${ }^{3}$ Department of Community Medicine, ${ }^{4}$ Department of Pathology, ${ }^{5}$ Kasturba Medical College, Manipal University, Karnataka Manipal, India *For correspondence: sabeenauthradam@ gmail.com,parvati.bhat@manipal.edu 
region and information about the epidemiologic data from every subpopulation is required prior any intervention like vaccine implementation (Nigam et al., 2014). In this context we decided to carry out a community study to detect genital HPV infection rate among rural women using urine samples.

\section{Materials and Methods}

\section{Study design}

A cross sectional study was carried out among asymptomatic rural women in the age group of 18-65 years of age during the period August 2013-May 2015 in a village in Udupi taluk with a population of 10,565 (Sabeena et al., 2015). Permission from Directorate of health and Family welfare services, Government of Karnataka was taken and was approved by Institutional Ethical Committee of Kasturba Medical College, Manipal University, and Manipal (IEC244/2013) before initiating the study. Collaboration with the local Primary Health care Centre and subcentre was established to reach the participants.

\section{Sample size and Sampling}

Minimum sample size of 704 married women was calculated with the assumption that the expected prevalence of HPV among married women as $12 \%$ at $95 \%$ confidence interval and $20 \%$ relative precision (Senapathy et al., 2011).

Inclusion criteria: Physically and mentally competent women in the age group of 18-65 who were permanent residents of Perdoor grampanchayath formed the study population and women willing to participate in the study were enrolled by house visits. Even unmarried women, pregnant women and women who had undergone hysterectomy for reasons other than cervical cancer were included.

\section{Subject recruitment}

The objective and public health importance of the study was explained to local government doctor, staff at Primary Health Centre and subcentre and to ASHA (Accredited Social Health Activists) workers. Households were visited with ASHA workers if at least one lady in the age group of 18-65 years was residing in that house. Before enrollment into the study all participants were given subject information sheet in local language (Kannada). Women who agreed to participate were asked to sign the written informed consent. Epidemiological data on age, occupation, literacy, socioeconomic status, diet, wood smoke exposure, tobacco exposure, alcohol consumption, marital history, reproductive aspects and genital hygiene were obtained from all enrolled women.

\section{Sample collection}

An early stream random urine sample was collected and transported to laboratory at $4-8^{\circ} \mathrm{C}$.

\section{Sample aliquoting:}

About $20-25 \mathrm{ml}$ urine sample collected per participant was mixed well and centrifuged at $3800 \mathrm{~g}$ for 20 minutes at $4^{\circ} \mathrm{C}$ in a refrigerated centrifuge (Sorvall Legend XTR Thermo Fisher Scientific, Germany).The middle opaque phase was collected, transferred into $1.5 \mathrm{ml}$ microcentrifuge tube and centrifuged at $16,000 \mathrm{~g}$ for 15 minutes at $4^{\circ} \mathrm{C}$. The pellets obtained from this were suspended in PBS, aliquoted and stored at $-70--80{ }^{\circ} \mathrm{C}^{\circ} \mathrm{C}$ (Tanzi et al., 2013). DNA was extracted using Qiagen viral DNA kit as per manufactures instructions.

\section{PCR inhibition by urine samples}

To check whether the urine sample is inhibiting PCR reaction, urine sample collected from a person not from the study area was spiked with a sample positive for HPV. Viral DNA was extracted from spiked sample and tested for HPV by standardized protocol. The sample was tested positive for HPV and the inhibition of PCR by urine was ruled out. This experiment validates the use of urine for detection of HPV in the study.

\section{HPV DNA testing}

Samples were tested for Human Papillomavirus (HPV) by nested PCR using PGMY09/11 and GP5+/6+ primer sets (R., 1995; Gravitt et al., 2000). Genotyping of positive cases was done by sequence analysis. Pap smear and cervical samples were taken from all consenting married women with HPV infection.

\section{Data analysis}

Descriptive statistics were reported using mean \pm SD for age and frequency and percentage for categorical variables. Statistical analysis was done using Epi Info7.

\section{Results}

From the study area 1324 women were approached by house visits of which 1307 agreed to participate in the study with a response rate of $98.7 \%$. All these women were personally interviewed and early stream random urine samples were collected. Two samples were lost during transportation and 1305 samples were aliquoted and tested by HPV nested PCR. Among the five urine samples tested positive for HPV DNA one sample could be typed as HPV-18. Only five urine samples were tested positive for HPV $(0.4 \%)$. As shown in Table-1, around half of the participants enrolled in the study were in the age group of

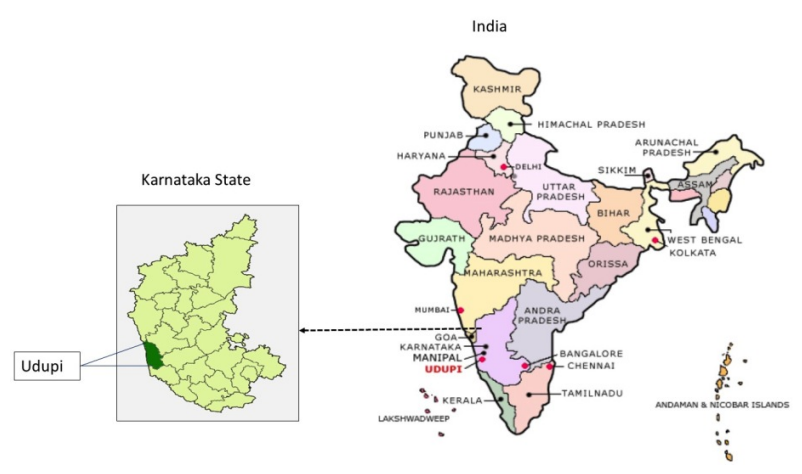

Figure 1. Geographical map showing study area in Udupi district, Karnataka, India 
Table 1. Sociodemographic characteristics of HPV study participants $(\mathbf{n}=\mathbf{1 3 0 5})$

\begin{tabular}{|c|c|c|}
\hline $\begin{array}{l}\text { Sociodemographic } \\
\text { characteristics }\end{array}$ & Number (\%) & $\begin{array}{l}\text { HPV DNA+ve } \\
\text { in urine samples }\end{array}$ \\
\hline \multicolumn{3}{|l|}{ Age groups(years) } \\
\hline $18-24$ & $31(12.4)$ & 0 \\
\hline $25-34$ & $323(24.8)$ & 1 \\
\hline $35-44$ & $355(27.2)$ & 2 \\
\hline $45-54$ & $307(23.5)$ & 1 \\
\hline $55-60$ & $198(15.2)$ & 1 \\
\hline $61-65$ & $91 \quad(6.9)$ & 0 \\
\hline \multicolumn{3}{|l|}{ Religion } \\
\hline Hindu & $1218(93.3)$ & 5 \\
\hline Muslim & $85(6.5)$ & 0 \\
\hline Christian & $2(0.2)$ & 0 \\
\hline \multicolumn{3}{|c|}{ Socioeconomic status } \\
\hline Low & $805(61.7)$ & 4 \\
\hline Middle & $489(37.5)$ & 1 \\
\hline High & $11(0.8)$ & 0 \\
\hline \multicolumn{3}{|l|}{ Literacy } \\
\hline Illiterate & $183(14.0)$ & 3 \\
\hline Primary & $210(16.1))$ & 0 \\
\hline Middle school & 364 (27.9) & 1 \\
\hline High school & $326(25.0)$ & 0 \\
\hline PUC & $117(9.0)$ & 0 \\
\hline Graduate & $105 \quad(8.1)$ & 1 \\
\hline \multicolumn{3}{|l|}{ Occupation } \\
\hline Home maker & $786(60.2)$ & 2 \\
\hline Unskilled & $379(29.0)$ & 0 \\
\hline Semiskilled & $12(1.0)$ & 0 \\
\hline Skilled & $94 \quad(7.3)$ & 3 \\
\hline Students & 34 (2.6) & 0 \\
\hline \multicolumn{3}{|c|}{ Age at marriage (1134) } \\
\hline$\leq 15$ & $25(2.2)$ & 0 \\
\hline $16-17$ & $58(5.1)$ & 0 \\
\hline $18-19$ & $187(16.5)$ & 2 \\
\hline$\geq 20$ & $864(76.2)$ & 3 \\
\hline
\end{tabular}

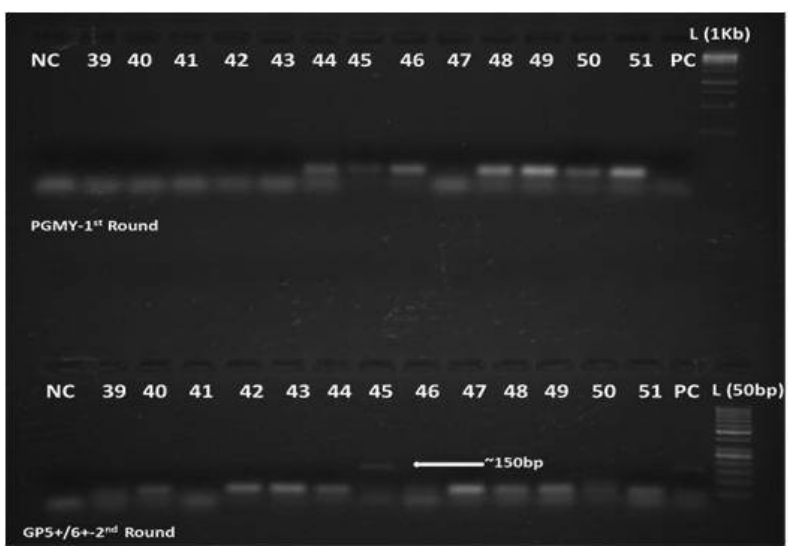

Figure 2. Gel Electrophoresis Picture Showing Nested HPV PCR Products

25-44 years (52.8\%). Mean age of the study participants was 38.8 years $(\mathrm{SD}=12.3)$ and mean age at marriage was found to be 22.1 years $(\mathrm{SD}=3.9)$. All the women detected to be infected with HPV were above 30 years of age including one 60 year old postmenopausal lady. Among the study population majority were Hindus $(93.3 \%)$ and $0.2 \%$ were Christians. According to modified Udai

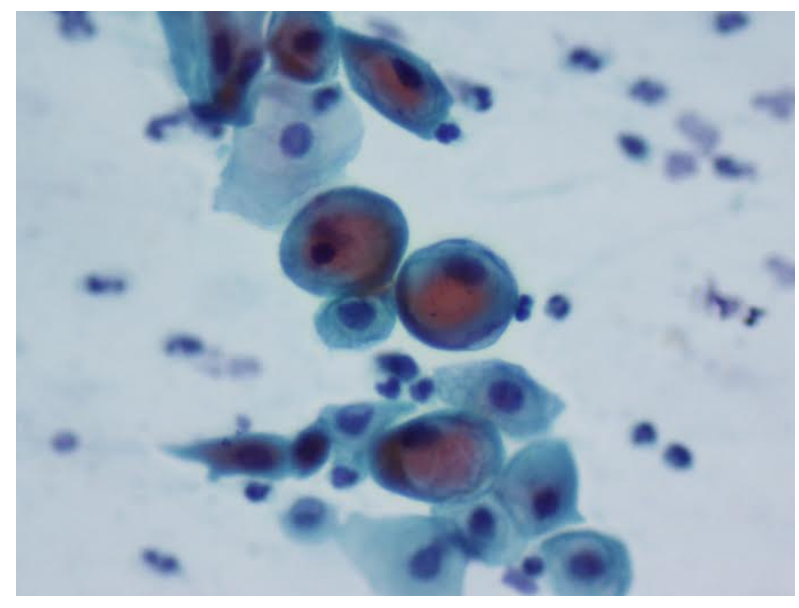

Figure 3. Cytology showing atypical squamous cells with nucleomegaly and perinuclear halo suggestive of HPV infection PAPx400

Pareek scale 805 women were from low socioeconomic status $($ score $<40)$ and 489 women were from middle socioeconomic status (score 40-70). A high literacy rate of $86.6 \%$ was noted among the study participants and most of them had received education at least up to middle class. Two-third of the women who participated in the study were home makers $(60.3 \%)$. Cashew factory workers constituted the major portion of working women and beedi rolling was done by $10.9 \%$ women at home.

Most of the families lived in mixed houses built of stones, cement and tiled roof $(80.7 \%)$. Eventhough almost all households had direct access to drinking water facility, running water facility inside house was reported by only 596 women (45.7\%) and this is often related to genital hygiene. After sexual contact 257 women (19.7\%) reported washing of genital areas. Majority of the study population were non-vegetarians $(94.3 \%)$ and more than $80 \%$ households used firewood for cooking. Age at marriage was 20 or above for three-fourth of the enrolled women. Only 24 years women had undergone Pap smear testing (1.8\%). Contact with multiple sexual partners was reported by a widow.

Women detected to be infected with HPV were called to village subcentre on a prefixed date. Pap smear and cervical samples were taken from four women after taking consent and all cervical samples were tested positive for HPV. Among the Pap smears collected three were normal smears. The fourth one collected from a widow with multiple partners was suggestive of Atypical Squamous Cells of Undermined Significance (ASC-US) as shown in Figure-3 and the cervical swab collected from the same participant was typed as HPV-18.

\section{Discussion}

Very low detection rate of genital HPV infection among rural women in the current study may be explained by the higher age at initiation of sexual activity and widely practiced monogamous sexual life style among both men and women. Mean age at marriage for Indian women is 21.6 years as per 2011 census, but half of the rural women and one third of the urban women in India are married 
between 18-20yearsof age. In Perdoor study two thirds of the study participants got married at or after 20 years of age. Data from a community based screening trial from Osmanabad district, Maharashtra, India conducted among women in the age group of 30-59 years reported a HPV prevalence of $10.3 \%$. But the mean age at first sexual contact was 15 years and high number of teenage pregnancies were reported among these women(Sauvaget et al., 2011). Immature transformation zone is mainly susceptible to HPV infection and younger age at sexual debut is a well-recognized risk factor for HPV infection (Veldhuijzen et al., 2010). In Perdoor study only one woman had multiple sexual partners and two women reported extramarital relations of their husbands. Almost all participants had single life time sexual partner. Multiple sexual partners increase the risk of incident as well as persistent HPV infections (Mollers et al., 2013). Women with 4 or more sexual partners were almost four times more at risk of having any HPV infection compared to women with single partner (Nahar et al., 2014).

The causal association of HPV with cervical cancer is well accepted and the incidence of cervical cancer cases can be related with HPV prevalence in that area. Cervical cancer is the second commonest cancer among women from this area and like most parts of India number of cervical cancers are coming down. There are no population data regarding asymptomatic genital HPV infection from this part of country. Recently published hospital based study from Udupi district found lower HPV prevalence of $82.5 \%$ among cervical cancer samples in contrast to previous Indian studies (Kabekkodu et al., 2015). Two recent studies from Central South China and Iran also reported HPV prevalence of $75.7 \%$ and $76 \%$ in cervical cancers respectively (Yang et al., 2014) (Jalilvand et al., 2015). A population based study conducted in Southern Iran revealed very low genital HPV prevalence of $0.6 \%$ and clinic based study from the same locality reported prevalence of 5\% among women. Among 799 women in the age group of 21-50 years participated in the Iran study cervical samples from only 5 pregnant women were tested positive (Eghbali et al., 2012).

HPV detection rate observed in our study is lower than what observed in previous Indian studies. Population studies from India report varying prevalence rates of $16.9 \%$ among women from Tamilnadu to $3.6 \%$ from Andaman Nicobar (Franceschi et al., 2005; Parvez et al.,2012; Sureshkumar et al., 2015). A study conducted using urine samples among tribal women in the age group of 9-25years from India found a higher HPV prevalence of $12.9 \%$ and more than two third of the participants were infected with High risk HPV (Sharma et al., 2015). Important factors to be considered is the age of study participants, younger age at sexual debut, widely practiced polygamy, home deliveries and multiparity among these indigenous subgroups and this data cannot be representative of entire India.

In the major population study from Dindigal, Tamilnadu, India home visits were paid to inform the rural women about the study and were invited to the clinic for vaginal examination (Franceschi et al., 2005). Higher refusal rate was reported in this study. Other two major population studies enrolled women from Gynecology clinics and cervical cancer screening programmes (Srivastava et al., 2012; Sureshkumar et al., 2015). Globally higher prevalence is seen in cancer screening clinics as most of the women might be having some gynecological problems and willingness for participation depends on their awareness and health seeking behavior. Women participating in voluntary cervical cancer screening programmes showed higher prevalence compared to population based survey conducted in Italy (Giorgi Rossi et al., 2010). Clinic based reporting of prevalence data should be adjusted downward according to population based surveys (Gouws et al., 2008). In our study asymptomatic women were included by house to house visits and urine samples were collected at home and transported to laboratory.

In developing countries cervical cancer is always associated with poverty, illiteracy and multiparity. These studies show that with literacy, better adoption of family planning practices and genital hygiene a reduction in HPV infection is achievable. Perdoor village in Udupi district shows a higher female literacy rate and better standards of living compared to the rest of India (Nair et al., 2000). Prevalence study carried out in North India found higher prevalence among rural women following non vegetarian diet and using homemade pads during menstruation (Srivastava et al., 2012). In our study $95 \%$ were following non vegetarian diet and three fourth of the study population used homemade pads.

Last two decades witnessed HPV detection studies using urine samples and it shows a sensitivity of $90 \%$ and specificity of $70 \%$ in detecting genital infection among women(O'Leary et al., 2011). Urine is an adequate alternative specimen to monitor HPV prevalence among women when there are cultural and religious hardships in obtaining genital samples (Vorsters et al., 2012) (Enerly et al., 2013) (Mendez et al., 2014). Eventhough urine samples are less sensitive compared to cervical samples, it is inexpensive and more socially acceptable for large epidemiological surveys in developing countries. Urine samples are moderately sensitive for detecting HPV and highly specific for detecting any HPV including HPV16 and 18 (Pathak et al., 2014). Urine based screening can reduce the number of false positive results and unnecessary invasive procedures. HPV detection in urine can be improved by using the first void urine (Vorsters et al., 2014). In our study women who had undergone hysterectomy for benign reasons were also included as there are case reports of HPV infection of vagina among hysterectomised women (D'Souza G, 2012). To the best of our knowledge this is the first population study conducted among asymptomatic women from Karnataka regarding genital HPV infection using urine samples. Another study conducted among the general population from North Karnataka using salivary samples found alarmingly high prevalence of HPV in the order of $64.3 \%$ and HPV-18 was the predominant type (Kulkarni et al., 2011). Study claimed to collect 369 salivary samples irrespective of sex and age of participants from rural and urban areas. When prevalence from a population group is discussed age, sex and immunity matters as HPV shows higher prevalence 
among adolescents and immunocompromised individuals.

All the women tested HPV-positive in Perdoor study were above 30 years of age including one 65 year old postmenopausal lady. Population studies from various parts of India found increased HPV prevalence as age advances (Sankaranarayanan et al., 2005; Asiaf et al.,2012; Srivastava et al., 2012). Highest HPV prevalence occurs soon after initiation of sexual activity among young women in the age group of 18-24 years and gradually comes down with a second peak after the fifth decade of life (Trottier et al., 2010). In this context WHO recommendation of screening of women 35 years or above and every five years for three tests in life time will be a more cost effective approach in resource poor settings (Aggarwal, 2014). In our study male condom usage was reported by few couples for spacing of pregnancies and male condoms protect from HPV infections partially (Moscicki et al., 2012). Smokeless tobacco use was found among few women in the form of chewing pan or nasal powder. Very few women were found to be taking alcoholic beverages. Tobacco and alcohol increase the risk of persistent High risk HPV infection and unsafe sexual practices (Veldhuijzen et al., 2010). Male circumcision rate in our study was $6.4 \%$ and according to meta-analysis study circumcision reduces HPV infection and high risk HPV prevalence among men (Albero et al., 2012). All the cervical samples collected from Perdoor women were HPV DNA positive showing $100 \%$ concordance. Another study by Prusty et al also found $100 \%$ agreement in HPV positivity between cervical and urine samples (Prusty et al., 2005). A metaanalysis study comparing the sensitivity and specificity of Pap smear and HPV DNA found better sensitivity and specificity of HPV-DNA testing in the order of $94.6 \%$ and $94.2 \%$ (Koliopoulos et al., 2007). Cervical cancer screening rate is very low among women from Karnataka and from this study area very few women had undergone Pap smear testing. Another factor to be considered is the fall in the incidence of both HPV infections and cervical cancer among women from the study area.

Strength of the study: This is the first major population study conducted among rural women from Karnataka to detect genital HPV infection using urine samples. We could screen 1305 rural women by house visits for genital HPV infection from an area from where, there was no previous community based study in this aspect using genitourinary samples. Modified aliquoting of urine samples was employed for better DNA extraction.

Limitation: Main limitation of our study was the nonprobability convenient sampling.

Research implications: Urine sampling enabled wider coverage of rural women including unmarried and pregnant women for whom genital sampling would have been a difficult option in the current study. This sampling strategy will be more acceptable to rural women with low screening compliance very low genital HPV prevalence was observed among rural asymptomatic women in the present study. studies.

In conclusion, Studies concerning HPV prevalence is required from different parts of India as there is vast social, linguistic and religious diversity across the country. Comprehensive data on prevalence of specific genotypes from various parts will be helpful in choosing appropriate prophylactic vaccines.

\section{Acknowledgements}

Financial support from ICMR project File no.5/8/7/15/2010/ECD-I. We also acknowledge the valuable help provided by Udupi District Health Officer Dr Rohini Junior Health Assistant Mrs.Vimala Bairy, ASHA workers Mrs.YashodaNaik, Mrs.Radha Bhat, Mrs Saraswathi Shetty, and Mrs Jayanthi.

\section{References}

Aggarwal P (2014). Cervical cancer: Can it be prevented? World J Clin Oncol, 5, 775-80.

Albero G, Castellsague X, Giuliano AR, et al (2012). Male circumcision and genital human papillomavirus: a systematic review and meta-analysis. Sex Transm Dis, 39, 104-13.

Asiaf A, Ahmad ST, Zargar MA, et al (2012). Prevalence of human papillomavirus infection in a Kashmiri ethnic female population. Genet Test Mol Biomarkers, 16, 904-9.

Bhatla N, Lal N, Bao YP, et al (2008). A meta-analysis of human papillomavirus type-distribution in women from South Asia: implications for vaccination. Vaccine, 26, 2811-7.

Bruni L, Diaz M, Castellsague X, et al (2010). Cervical human papillomavirus prevalence in 5 continents: meta-analysis of 1 million women with normal cytological findings. J Infect Dis, 202, 1789-99.

D'Souza G BR, Zhong Y, Minkoff H, Massad LS, Xue X, et al. (2012). Cervicovaginal HPV infection before and after hysterectomy: evidence of different tissue tropism for oncogenic and non-oncogenic HPV types in a cohort of HIV-positive and HIV-negative women. Int J Cancer J Int Cancer, 131, 1472-8. .

Das B.C SJK, Gopalkrishna. V, Das D.K, Singh V, Gissmann L, et al (1992). A high frequency of human papillomavirus DNA sequences in cervical carcinomas of Indian women as revealed by southern blot hybridization and polymerase chain reaction. $J$ Med Virol, 36, 239-45.

Eghbali SS, Amirinejad R, Obeidi N, et al (2012). Oncogenic human papillomavirus genital infection in southern Iranian women: population-based study versus clinic-based data. Virol J,9, 194.

Enerly E, Olofsson C, Nygard M (2013). Monitoring human papillomavirus prevalence in urine samples: a review. Clin Epidemiol, 5, 67-79.

Franceschi S, Rajkumar R, Snijders PJ, et al (2005). Papillomavirus infection in rural women in southern India. Br J Cancer, 92, 601-6.

Giorgi Rossi P, Bisanzi S, Paganini I, et al (2010). Prevalence of HPV high and low risk types in cervical samples from the Italian general population: a population based study. $B M C$ Infect Dis, 10, 214.

Gouws E, Mishra V, Fowler TB (2008). Comparison of adult HIV prevalence from national population-based surveys and antenatal clinic surveillance in countries with generalised epidemics: implications for calibrating surveillance data. Sex Transm Infect, 84, 17-23.

Gravitt PE, Peyton CL, Alessi TQ, et al (2000). Improved amplification of genital human papillomaviruses. J Clin Microbiol, 38, 357-61.

Jalilvand S, Shoja Z, Nourijelyani K, et al (2015). Meta-analysis of type-specific human papillomavirus prevalence in Iranian women with normal cytology, precancerous cervical lesions and invasive cervical cancer: Implications for screening and 
vaccination. J Med Virol, 87, 287-95.

Kabekkodu SP, Bhat S, Pandey D, et al (2015). Prevalence of human papillomavirus types and phylogenetic analysis of HPV-16 L1 variants from Southern India. Asian Pac J Cancer Prev, 16, 2073-80.

Koliopoulos G, Arbyn M, Martin-Hirsch P, et al (2007). Diagnostic accuracy of human papillomavirus testing in primary cervical screening: a systematic review and metaanalysis of non-randomized studies. Gynecol Oncol, 104, 232-46.

Kulkarni SS, Kulkarni SS, Vastrad PP, et al (2011). Prevalence and distribution of high risk human papillomavirus (HPV) Types 16 and 18 in Carcinoma of cervix, saliva of patients with oral squamous cell carcinoma and in the general population in Karnataka, India. Asian Pac J Cancer Prev, 12, 645-8

Mendez K, Romaguera J, Ortiz AP, et al (2014). Urine-based human papillomavirus DNA testing as a screening tool for cervical cancer in high-risk women. Int J Gynaecol Obstet, 124, 151-5.

Mollers M, Boot HJ, Vriend HJ, et al (2013). Prevalence, incidence and persistence of genital HPV infections in a large cohort of sexually active young women in the Netherlands. Vaccine, 31, 394-401.

Moscicki AB, Schiffman M, Burchell A, et al (2012). Updating the natural history of human papillomavirus and anogenital cancers. Vaccine, 30, 24-33.

Nahar Q, Sultana F, Alam A, et al (2014). Genital human papillomavirus infection among women in Bangladesh: findings from a population-based survey. PLoS One, 9, 107675 .

Nair NS, Rao RS, Chandrashekar S, et al (2000). Sociodemographic and maternal determinants of low birth weight: a multivariate approach. Indian J Pediatr, 67, 9-14.

Nigam A, Saxena P, Acharya AS, et al (2014). HPV vaccination in India: critical appraisal. ISRN Obstet Gynecol, 2014, 394595.

O’Leary MC, Sinka K, Robertson C, et al (2011). HPV typespecific prevalence using a urine assay in unvaccinated male and female 11- to 18-year olds in Scotland. Br J Cancer, 104, 1221-6.

Parvez R, Hedau S, Bhattacharya D, et al (2012). High-risk HPV infection among the tribal and non-tribal women of the andaman and nicobar islands, India. Public Health, 126, 67-9.

Pathak N, Dodds J, Zamora J, et al (2014). Accuracy of urinary human papillomavirus testing for presence of cervical HPV: systematic review and meta-analysis. BMJ, 349, 5264 .

Prusty BK, Kumar A, Arora R, et al (2005). Human papillomavirus (HPV) DNA detection in self-collected urine. Int J Gynaecol Obstet, 90, 223-7.

R. dRA-Md (1995). The use of general primers GP5+/ GP6+elongated at their 3' ends with adjacent highly conserved sequences improves human papilloma virus detection by PCR. J Gen Virol, 76, 1057-62.

Sabeena S, Bhat PV, Kamath V, et al (2015). Knowledge, attitude and practice concerning human papilloma virus infection and its health effects among rural women, Karnataka, South India. Asian Pac J Cancer Prev, 16, 5053-8.

Sankaranarayanan R, Nene BM, Dinshaw KA, et al (2005). A cluster randomized controlled trial of visual, cytology and human papillomavirus screening for cancer of the cervix in rural India. Int J Cancer, 116, 617-23.

Sauvaget C, Nene BM, Jayant K, et al (2011). Prevalence and determinants of high-risk human papillomavirus infection in middle-aged Indian women. Sex Transm Dis, 38, 902-6.

Senapathy JG, Umadevi P, Kannika PS (2011). The present scenario of cervical cancer control and HPV epidemiology in India: an outline. Asian Pac J Cancer Prev, 12, 1107-15.

Sharma K, Kathait A, Jain A, et al (2015). Higher prevalence of human papillomavirus infection in adolescent and young adult girls belonging to different Indian tribes with varied socio-sexual lifestyle. PLoS One, 10, 125693.

Srivastava S, Gupta S, Roy JK (2012). High prevalence of oncogenic HPV-16 in cervical smears of asymptomatic women of eastern Uttar Pradesh, India: a population-based study. J Biosci, 37, 63-72.

Sureshkumar BT, Shanmughapriya S, Das BC, et al (2015). A population-based study of the prevalence of HPV in three districts of Tamil Nadu, India. Int J Gynaecol Obstet, 129, 58-61.

Tanzi E, Bianchi S, Fasolo MM, et al (2013). High performance of a new PCR-based urine assay for HPV-DNA detection and genotyping. J Med Virol, 85, 91-8.

Trottier H, Ferreira S, Thomann P, et al (2010). Human papillomavirus infection and reinfection in adult women: the role of sexual activity and natural immunity. Cancer Res, 70, 8569-77.

Veldhuijzen NJ, Snijders PJ, Reiss P, et al (2010). Factors affecting transmission of mucosal human papillomavirus. Lancet Infect Dis, 10, 862-74.

Vorsters A, Micalessi I, Bilcke J, et al (2012). Detection of human papillomavirus DNA in urine. A review of the literature. Eur J Clin Microbiol Infect Dis, 31, 627-40.

Vorsters A, Van den Bergh J, Micalessi I, et al (2014). Optimization of HPV DNA detection in urine by improving collection, storage, and extraction. Eur J Clin Microbiol Infect Dis, 33, 2005-14.

Walboomers JM, Jacobs MV, Manos MM, et al (1999). Human papillomavirus is a necessary cause of invasive cervical cancer worldwide. J Pathol, 189, 12-9.

Yang L, Yang H, Wu K, et al (2014). Prevalence of HPV and variation of HPV 16/HPV 18 E6/E7 genes in cervical cancer in women in South West China. J Med Virol, 86, 1926-36. 